\title{
The Osteogenesis Effect and Underlying Mechanisms of Local Delivery of gAPN in Extraction Sockets of Beagle Dogs
}

\author{
Hongcheng Hu ${ }^{1,2, \dagger}$, Yinfei Pu ${ }^{1,2, \dagger}$, Songhe Lu ${ }^{1,2, \dagger}$, Kuo Zhang ${ }^{3}$, Yuan Guo ${ }^{1,2}$, Hui Lu ${ }^{1,2}$, \\ Deli Li ${ }^{1,2}$, Xuefen $\mathrm{Li}^{2}$, Zichen $\mathrm{Li}^{4}$, Yuwei Wu ${ }^{1,2, *}$ and Zhihui Tang ${ }^{1,2, *}$
}

1 2nd Dental Center, Peking University School and Hospital of Stomatology, Beijing 100101, China; E-Mails: carefree36@gmail.com (H.H.); puyinfei@yeah.net (Y.P.); tongtong20034@163.com (S.L.); gy2hcp@163.com (Y.G.); cute_luhui@yeah.net (H.L.); leedelee@163.com (D.L.)

2 National Engineering Laboratory for Digital and Material Technology of Stomatology, Peking University School and Hospital of Stomatology, Beijing 100081, China;

E-Mail: xflee7@163.com

3 Department of Laboratory Animal Science, Peking University Health Science Center, Beijing 100191, China; E-Mail: zhangkuo@bjmu.edu.cn

4 Department of Polymer Science \& Engineering College of Chemistry \& Molecular Engineering, Peking University, Beijing 100871, China; E-Mail: zcli@pku.edu.cn

$\dagger$ These authors contributed equally to this work.

* Authors to whom correspondence should be addressed;

E-Mails: kqwuyuwei@126.com (Y.W.); tang_zhihui@live.cn (Z.T.);

Tel.: +86-10-8219-6267 (Y.W.); +86-10-8219-6217 (Z.T.);

Fax: +86-10-6490-7970 (Y.W. \& Z.T.).

Academic Editor: Wenbin Deng

Received: 5 August 2015 / Accepted: 13 October 2015 / Published: 20 October 2015

\begin{abstract}
A plastic and biodegradable bone substitute consists of poly (L-lactic- $c o$-glycolic) acid and $30 \mathrm{wt} \% \beta$-tricalcium phosphate has been previously fabricated, but its osteogenic capability required further improvement. We investigated the use of globular adiponectin (gAPN) as an anabolic agent for tissue-engineered bone using this scaffold. A qualitative analysis of the bone regeneration process was carried out using $\mu \mathrm{CT}$ and histological analysis 12 weeks after implantation. CBCT (Cone Beam Computed Tomography) superimposition was used to characterise the effect of the different treatments on bone formation. In this study, we also explored adiponectin's (APN) influence on primary cultured human jaw bone marrow mesenchymal stem cells gene expressions involved in the osteogenesis. We found
\end{abstract}


that composite scaffolds loaded with gAPN or bone morphogenetic protein 2 (BMP2) exhibited significantly increased bone formation and mineralisation following 12 weeks in the extraction sockets of beagle dogs, as well as enhanced expression of osteogenic markers. In vitro investigation revealed that $\mathrm{APN}$ also promoted osteoblast differentiation of primary cultured human jaw bone marrow mesenchymal stem cells (h-JBMMSCs), accompanied by increased activity of alkaline phosphatase, greater mineralisation, and production of the osteoblast-differentiated genes osteocalcin, bone sialoprotein and collagen type I, which was reversed by APPL1 siRNA. Therefore, the composite scaffold loaded with APN exhibited superior activity for guided bone regeneration compared with blank control or Bio-Oss ${ }^{\circledR}$ (a commercially available product). The composite scaffold with APN has significant potential for clinical applications in bone tissue engineering.

Keywords: adiponectin; BMP2 (bone morphogenetic protein 2); dental material; mesenchymal stem cells; beagle dog

\section{Introduction}

We previously established a biodegradable scaffold composed of poly (L-lactic-co-glycolic) acid (PLGA), $\beta$-tricalcium phosphate ( $\beta$-TCP) and globular adiponectin (gAPN) $[1,2]$. This scaffold is particularly attractive for clinical applications due to its excellent biocompatibility and tunable biodegradation rate. The constituents are readily obtainable from synthetic resources in large quantities, and to enhance the functionality of the synthetic materials, peptide or protein ligands such as bone morphogenetic protein 2 (BMP2), which plays a crucial role in osteoinduction [3], can be used for molecular decoration. However, certain issues need to be addressed to facilitate its widespread practical application, including its complicated synthesis, easy degradation, and high cost [3-5]. Biological safety questions still need to be coped with, along with issues such as symptomatic ectopic bone formation, bone resorption or remodelling at the recipient region, as well as other possible complications including tumorigenesis and teratogenesis [4,5].

Adiponectin (APN) is the most abundant adipokine in blood plasma; the trimeric form of globular APN (gAPN), results from proteolytic cleavage of full-length APN (fAPN) [6,7]. APN harbors well-characterised insulin-sensitising, anti-inflammatory, and anti-atherosclerotic, as well as anti-diabetic properties [8,9]. More importantly, systemic APN infusion ameliorates diabetic mobilopathy of bone marrow mesenchymal stem cells (BMSCs), lowers glucose concentration, and promotes bone formation in mice with obesity [10]. A wide variety of studies have demonstrated that APN promotes bone formation via several mechanisms, including directly signalling osteoblasts to promote differentiation [11,12], favouring BMSC differentiation toward osteoblastic lineage [13-16], decreasing sympathetic tone [16,17], and inducing the production of BMP-2 in osteoblasts [18]. However, the role of APN in promoting osteogenic commitment of orofacial bone-derived BMSCs to enhance bone formation has not yet been probed and reported. This constitutes a novel approach for locally promoting bone formation by APN through targeting the osteoblasts and osteoclasts simultaneously. 
We conducted a comparative study of osteogenesis in scaffolds loaded with BMP2, gAPN, or Bio-Oss $^{\circledR}$ (a commercially available product that is routinely and successfully used in surgical dentistry and orthopaedic surgery), through the placement of biomaterials in the fresh extraction sockets of beagle dogs. We also experimentally investigated how gAPN affects osteoblast differentiation of human jaw bone marrow mesenchymal stem cells (h-JBMMSCs) in vitro. We hypothesised that scaffolds loaded with gAPN would enhance bone formation through promoting osteogenic commitment of h-JBMMSCs.

\section{Results}

\subsection{Characterization of Bone Substitutes Loaded with gAPN or BMP2}

Figure 1a demonstrates the morphology of chitosan microspheres with gAPN. The average diameter was $18.59 \pm 15.20 \mu \mathrm{m}$ (Figure 1a). The microstructure of the porous $\beta$-TCP is shown in Figure $1 \mathrm{~b}$ (pore size 100-600 $\mu \mathrm{m}$ ). The appearance of PLGA scaffold was a block mass (Figure 1c).

a

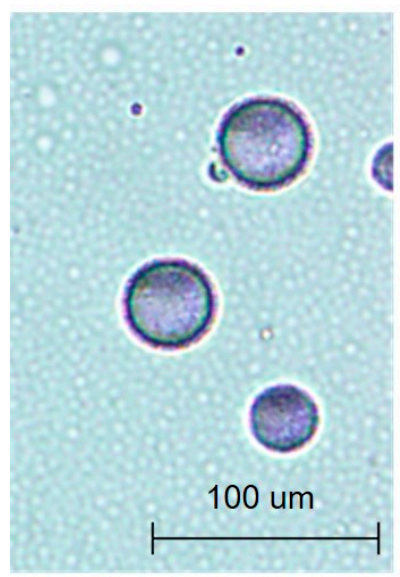

b

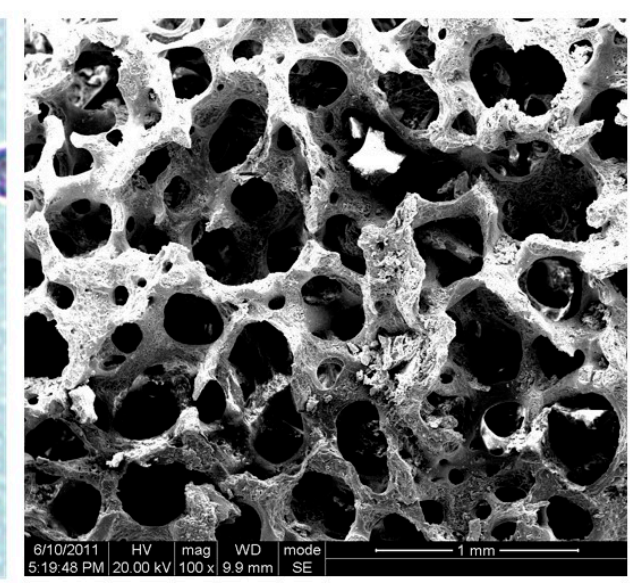

C

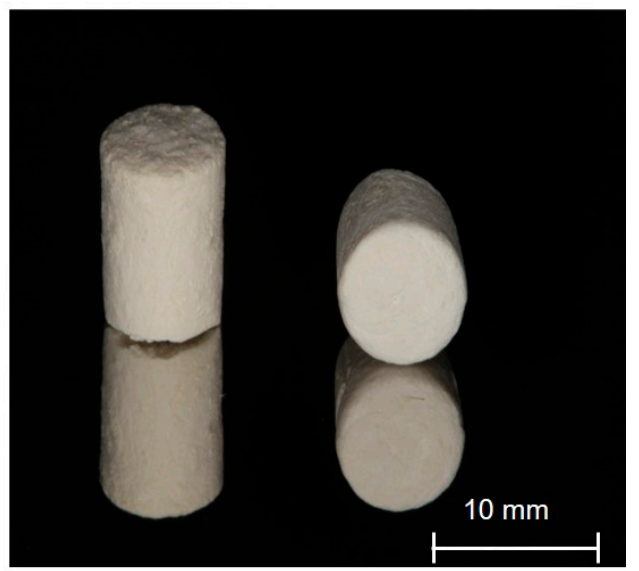

Figure 1. Optical microscopic (Nikon, Berlin, Germany) image ((a) scale bars $=100 \mu \mathrm{m})$ of chitosan microspheres with gAPN (blue purple); SEM image of the $\beta$-TCP scaffold ((b) scale bars $=1 \mathrm{~mm}$, particle size $0.25-1 \mathrm{~mm}$, pore size $100-600 \mu \mathrm{m}$ ) prepared in the PLGA (poly L-lactic-co-glycolic acid) scaffold. The appearance of PLGA scaffold ((c) scale bars $=10 \mathrm{~mm})$.

\subsection{Clinical Evaluation}

All sites healed without complications, and were observed daily during routine post-surgery checks. We observed full coverage of each site with a slightly inflamed mucosa during removal of the sutures after two weeks. At the time of sacrifice (12 weeks), all extraction sites were covered by an apparently non-inflamed keratinised ridge mucosa, and the outline of the extraction socket were difficult to identify. The socket distribution and bone fill were observed radiographically using the superimposed models, as shown in Figure 2. 

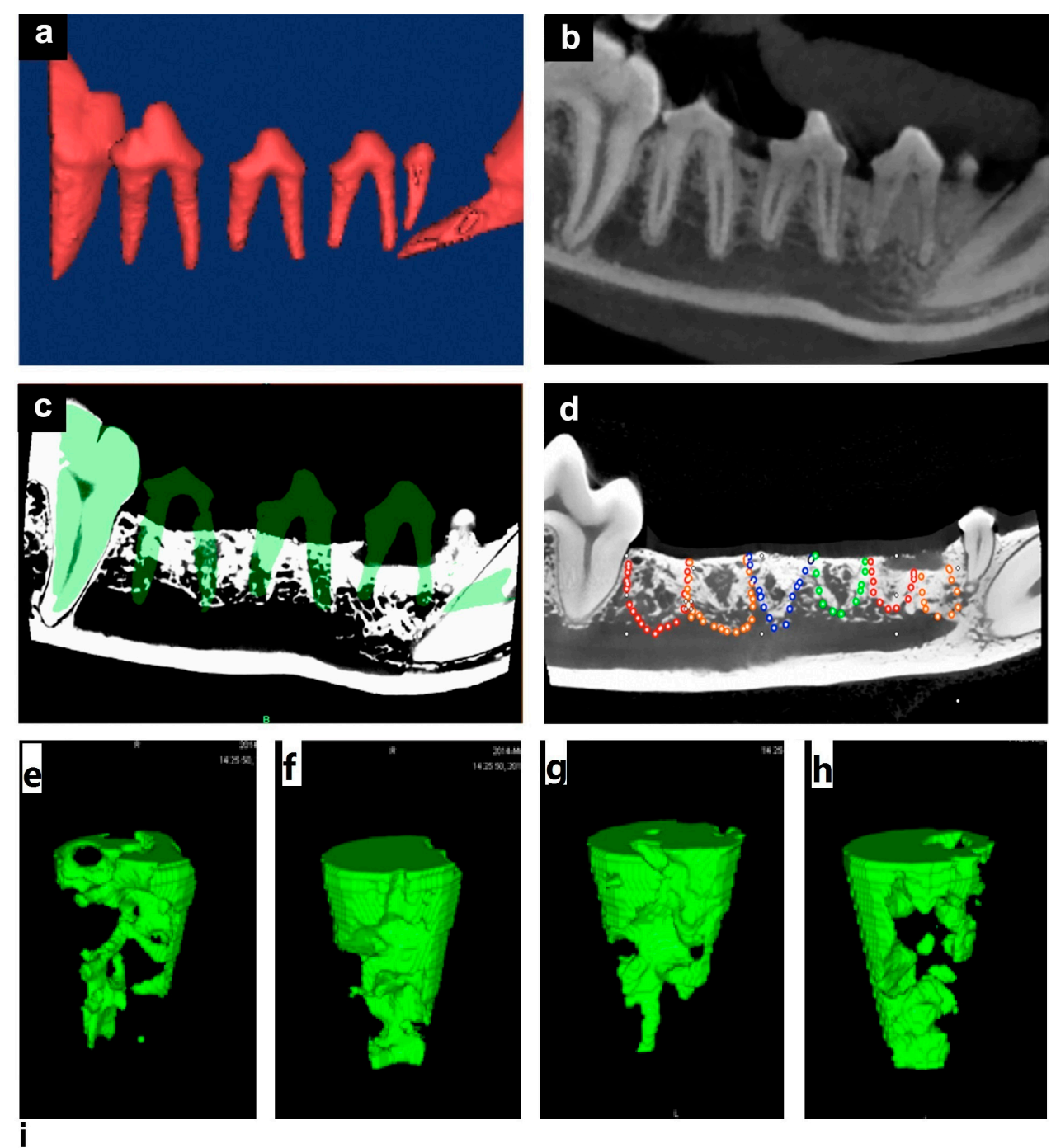

\begin{tabular}{cllll}
\hline & BV/TV & Thickness & Number & Seperation \\
\hline Blank & $1.00 \pm 0.06 *$ & $1.00 \pm 0.02 *$ & $1.00 \pm 0.03 *$ & $1.00 \pm 0.19 *$ \\
Bio-oss & $2.67 \pm 0.06$ & $0.87 \pm 0.02$ & $3.07 \pm 0.03$ & $0.44 \pm 0.19$ \\
gAPN & $1.25 \pm 0.06$ & $1.11 \pm 0.01$ & $1.13 \pm 0.03$ & $0.77 \pm 0.14$ \\
BMP2 & $1.36 \pm 0.06$ & $1.12 \pm 0.02$ & $1.24 \pm 0.03$ & $0.65 \pm 0.14$ \\
\hline
\end{tabular}

Figure 2. Extraction socket location and $\mu \mathrm{CT}$ analysis: (a) 3D CBCT (Cone Beam Computed Tomography) reconstructions and (b) the teeth used for reference; (c) Superimposition 3D CBCT and $\mu \mathrm{CT}$ models; (d) Highlighted borderline of the extraction socket in the $\mu \mathrm{CT}$ models (red: gAPN-treated group, orange: BMP2-treated group, blue: Bio-Oss group, green: blank group). Representative 3D reconstruction of the trabecular structure in the extraction socket: (e) Blank; (f) Bio-oss; (g) gAPN and (h) BMP2; and (i) Changes in trabecular bone parameters, including trabecular bone volume normalised by tissue volume (BV/TV), trabecular thickness (Tb.Th), trabecular number (Tb.N), and trabecular separation (Tb.Sp) are shown as means $\pm \mathrm{SD}$, with $n=4$. $^{*} p<0.05$, as determined by one-way ANOVA. 


\subsection{Bone Substitutes Loaded with gAPN or BMP2 Promoted Bone Formation}

To investigate the composites loaded with gAPN or BMP2 in bone metabolism, we delivered gAPN or BMP2 via the PLGA scaffold into the dogs' extraction sockets. We then used $\mu \mathrm{CT}$ (see Figure 2) and hematoxylin and eosin (H\&E) staining (see Figure 3) to analyse changes in the trabecular bone (Tb) structure and mineral density following 12 weeks of scaffold placement. Compared with blank controls, after 12 weeks in extraction sockets, the gAPN and BMP2 scaffolds led to a significant improvement in the trabecular bone volume (BV), trabecular thickness (Tb.Th), trabecular number (Tb.N), and to a reduction in the trabecular separation (Tb.Sp) distance (see Figure 2i). However, the Bio-Oss group exhibited the largest ratio of trabecular BV normalised by tissue volume (BV/TV) and Tb.N, as well as the lowest Tb.Sp (see Figure 2i). H\&E staining further demonstrated the ability of gAPN and BMP2 (see Figure 3) scaffolds to increase the trabecular bone mass and trabecular separation of the extraction sockets. No major bone formations were evident in the central region of the extraction sockets in the control group (see Figure 3).

As shown in Figure 3, all extraction sockets demonstrated trabecular bone fill with a more or less developed bone marrow after eight weeks healing. The anorganic bovine bone was with surrounded by lamellar bone and granulation tissue in the Bio-Oss group. In contrast, the extraction sockets treated with scaffolds loaded with gAPN or BMP2 exhibited pronounced resorption over the eight weeks and the newly formed trabecular bone apically and laterally extended from the socket walls, occupying almost the entire socket.

Compared with the control and Bio-Oss groups, the extraction sockets treated with scaffolds loaded with gAPN or BMP2 exhibited increase in the new bone formation, as shown by the increased toluidine blue staining images in Figure 4. We observed abundant newly formed osteoid in the supposed root area; however, in the Bio-Oss group the osteoid exhibited closer proximity and contact with the demineralised bovine granule, as shown by the slightly lighter staining than the osteoid. Thus, the new bone formation in the gAPN or BMP2 loaded group was superior to that of the control and Bio-Oss group (Figure 4).

Masson staining was also used to observe bone formation. In the blank control, only a thin layer of lamellar trabecular was observed, and the largest area was bone marrow, as shown in Figure 5a. Newly formed trabecular was observed hailed from the edges of the extraction socket toward the central area in the control group. In this histological sections, only a small volume of blue ossein was distributed on the new bone surface, and less was observed than that in host bone. In the Bio-Oss group, the central regions were mainly occupied by mineral portions of bovine bone. The implanted bovine bone had not been replaced, and the scaffold surface was surrounded by little osteoid, as shown in Figure 5b. In the gAPN and BMP2 treatment groups, the implanted scaffold had been replaced, and the treated defect was almost covered by mature lamellar bone, as shown by the red staining, and the blue ossein was spreaded consistently in the new bone matrix (see Figure 5c,d). These results further demonstrate that the gAPN and BMP2 effectively enhanced the trabecular bone density and mineralisation after 12 weeks of socket placement. 

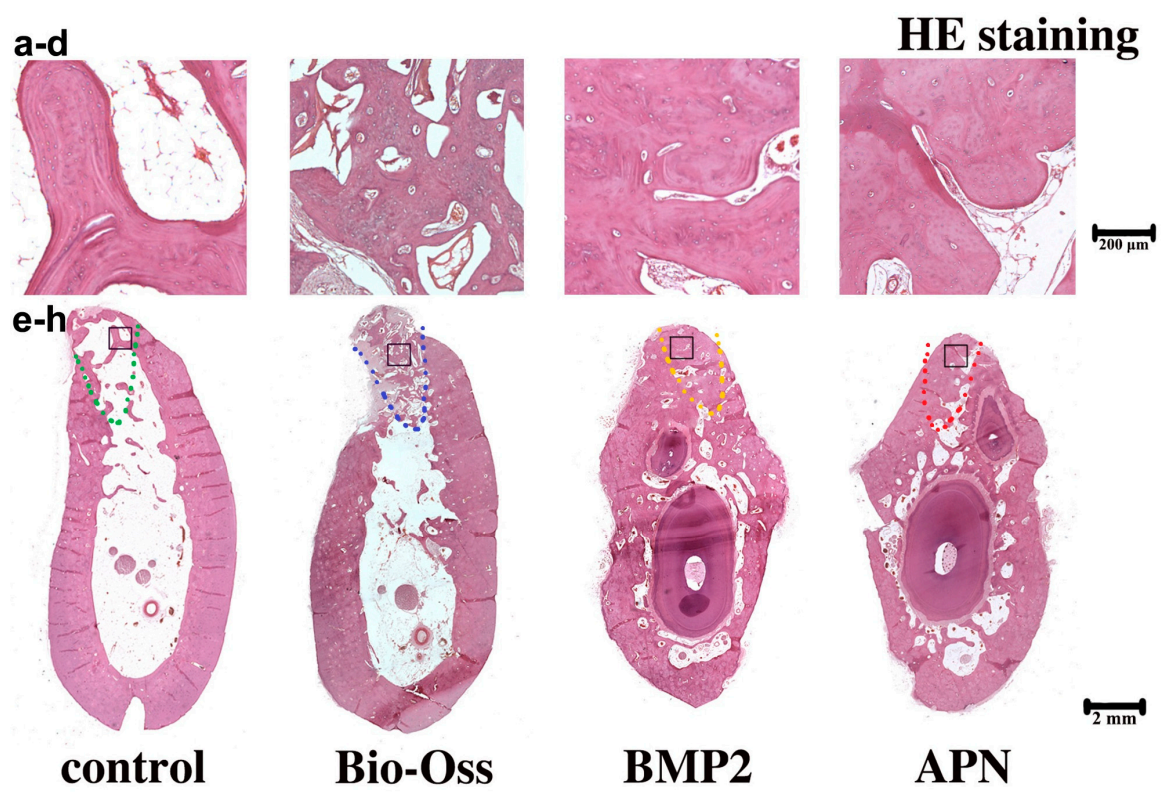

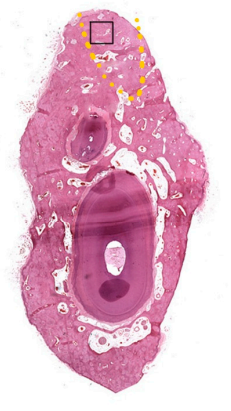

BMP2

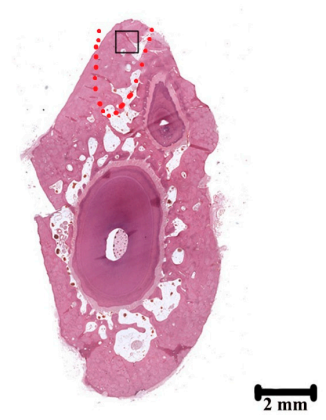

APN

Figure 3. Decalcified $H \& E$ staining of the extraction sockets following the different 12-week treatments: (a)The magnifying control group; (b) Bio-Oss group; (c) BMP2 group; (d) APN group; (e-h) represent the integral extraction socket of control group (e); Bio-Oss group (f); BMP2 group (g); APN group (h). The black square represents the magnifying area. The dashed line represents the extraction sockets. Magnification: upper panel $40 \times$; lower panel $4 \times . n=4$ for each group.

a-d

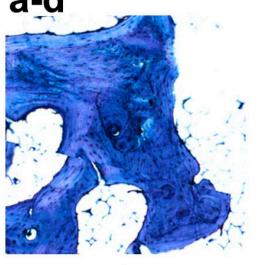

e-h

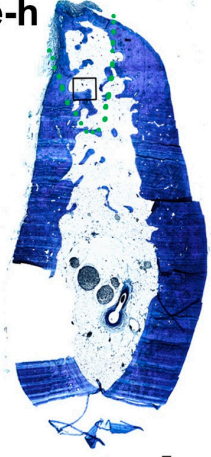

control
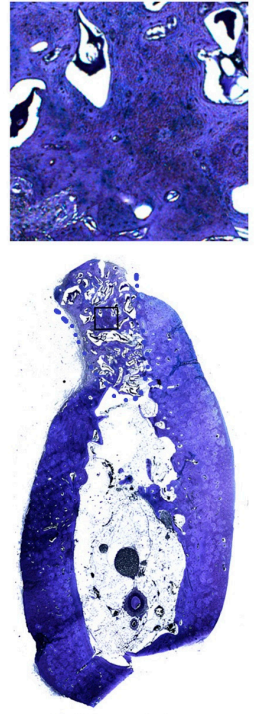

Bio-Oss
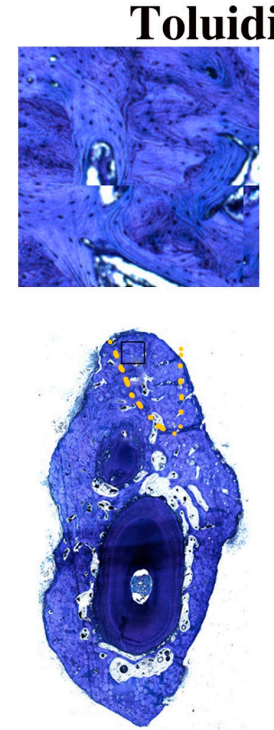

BMP2
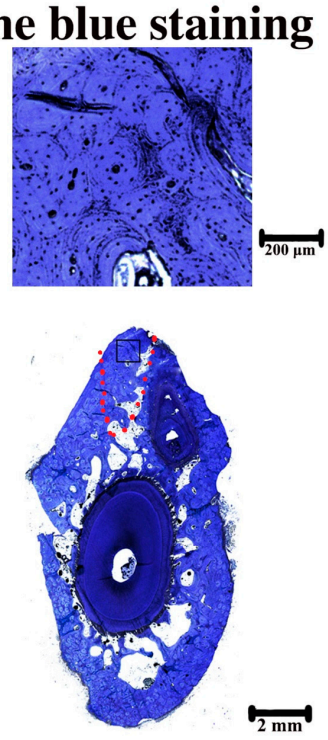

APN

Figure 4. Decalcified toluidine blue staining of extraction sockets following the different 12-week treatments: (a)The magnifying control group; (b) Bio-Oss group; (c) BMP2 group; (d) APN group; (e-h) represent the integral extraction socket of control group (e); Bio-Oss group (f); BMP2 group (g); APN group (h). The black square represents the magnifying area. The dashed line represents the extraction sockets. Magnification: upper panel $40 \times$; lower panel $4 \times . n=4$ for each group. 


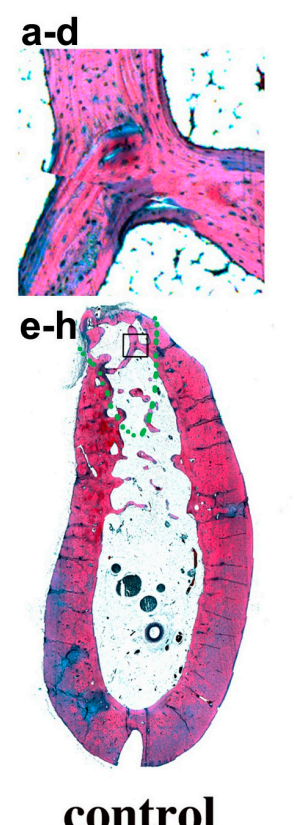

control

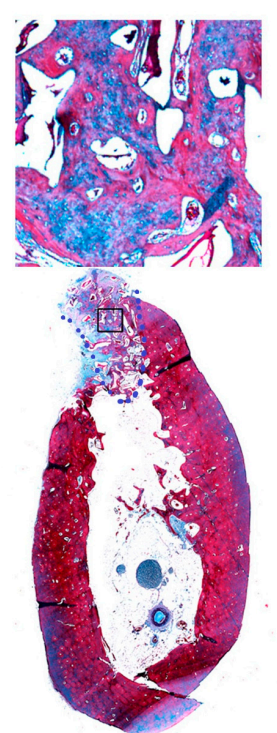

Bio-Oss

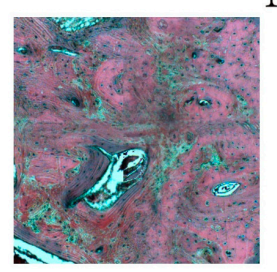

Masson staining

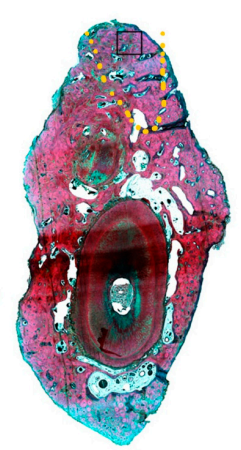

BMP2
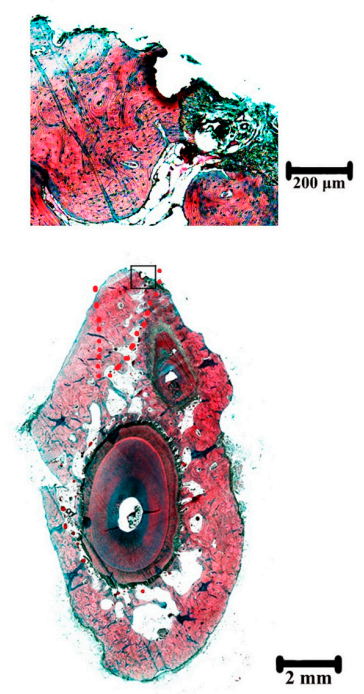

APN

Figure 5. Decalcified toluidine Masson staining of extraction sockets following the different 12-week treatments: (a) The magnifying control group; (b) Bio-Oss group; (c) BMP2 group; (d) APN group; (e-h) represent the integral extraction socket of control group (e); Bio-Oss group (f); BMP2 group (g); APN group (h). The black square represents the magnifying area. The dashed line represents the extraction sockets. New new bone is shown in red. Magnification: upper panel $40 \times$; lower panel $4 \times . n=4$ for each group.

\section{4. gAPN or BMP2 Treatment Increased Osteoblast Differentiation}

We characterised the expression in osteoblast-related genes by immunostaining. The extraction sockets treated with gAPN and BMP2 exhibited significantly increased osteocalcin and BSP expression compared with the control group, as shown in Figure 6a,b, Table S1. The expression of collagen type-I, which is a key scaffold and template for mineral formation [19], was significantly increased in the gAPN or BMP2 groups compared with the control, as shown in Figure 6c,d, Table S1. The expression of collagen in Bio-Oss group, however, was lower than in the other three groups.

\section{5. gAPN Promoted Osteogenic Differentiation of h-JBMMSCs (Human Jaw Bone Marrow Mesenchymal Stem Cells)}

h-JBMMSCs have the potential to differentiate into osteoblasts, chondrocytes, and adipocytes [20]. We found that the administration of gAPN promoted bone formation in extraction defects more actively than in the control and Bio-Oss groups, so we investigated the role of gAPN in regulating osteogenic commitment of h-JBMMSCs. The identified h-JBMMSCs were treated with $1 \mu \mathrm{g} / \mathrm{mL}$ of gAPN, and osteogenesis was evaluated using alizarin bordeaux staining, as shown in Figure 7a,b. We observed more positive alizarin bordeaux staining compared with control cells in the gAPN-treated cells, as shown in Figure 7c. Additionally, mRNA levels of ALP, BSP, Col-1, OCN, and OPN were significantly enhanced in the gAPN-treated cells compared with the control, as shown in Figure $7 \mathrm{~d}$. 
To further explore the mechanisms underlying the gAPN-induced osteogenic differentiation of the h-JBMMSCs, we evaluated the levels of APPL1 expression. We found that APPL1 expression increased significantly following gAPN treatment compared with the untreated control, as shown in Figure 7d. Additionally, we pretreated the h-JBMMSCs with the specific APPL1 siRNA (see Figure 7d), and found that downregulation of APPL1 resulted in downregulation of ALP, BSP, Col-1, OCN, and OPN.
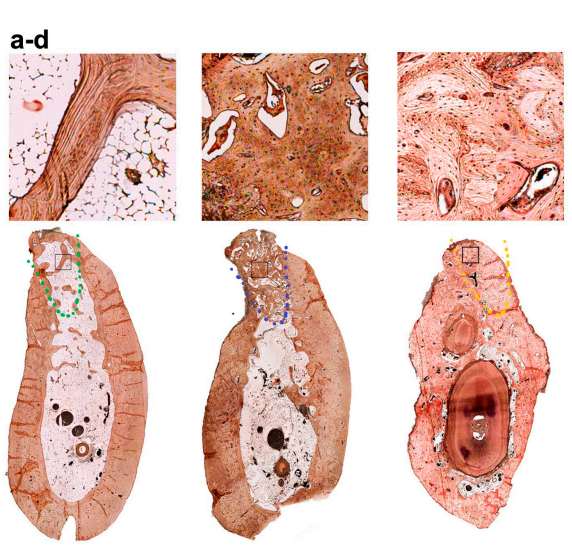

osteocalcin
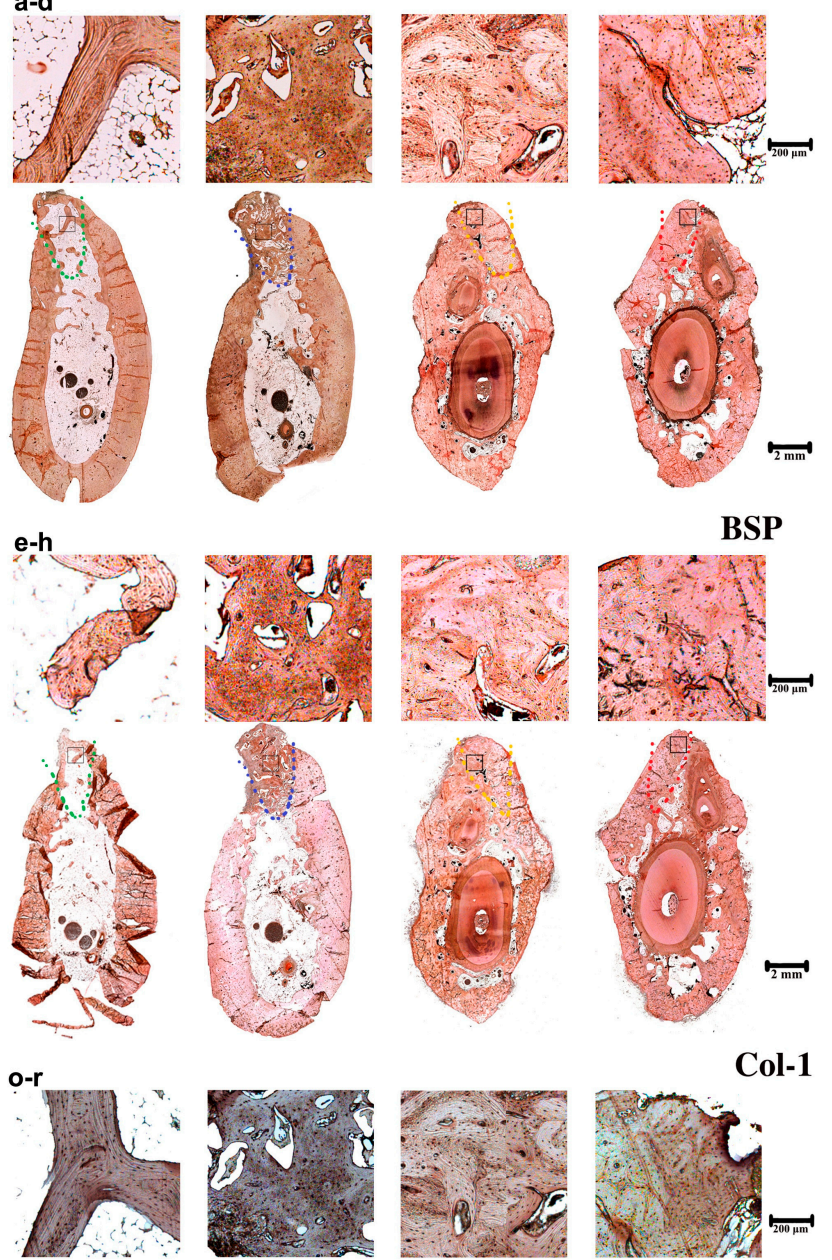

Col-1
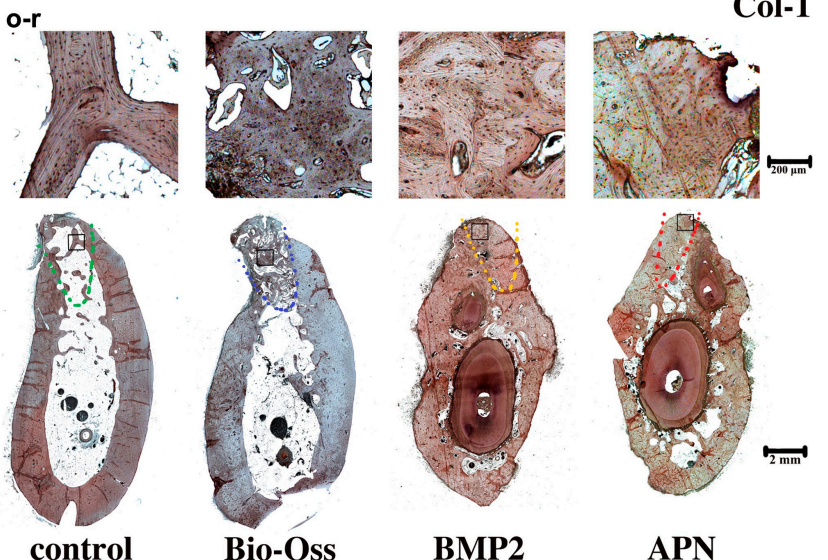

BMP2

APN

Figure 6. Expression of osteogenic related protein following the different 12-week treatments in the extraction sockets. Imunohistochemical analysis of extraction socket trabecular bone sections for (a-d) OCN (Osteocalcin); (e-h) BSP (Bone sialoprotein) and (o-r) Col-1 (Collagen 1). (a,e,o) the control group; (b,f,p) Bio-Oss group; (c,g,q) BMP2 group; $(\mathbf{d}, \mathbf{h}, \mathbf{r})$ APN group. The black square represents the magnifying area. The dashed line represents the extraction sockets. Magnification: upper panel 40×; lower panel $4 \times$. $n=4$ for each group. 

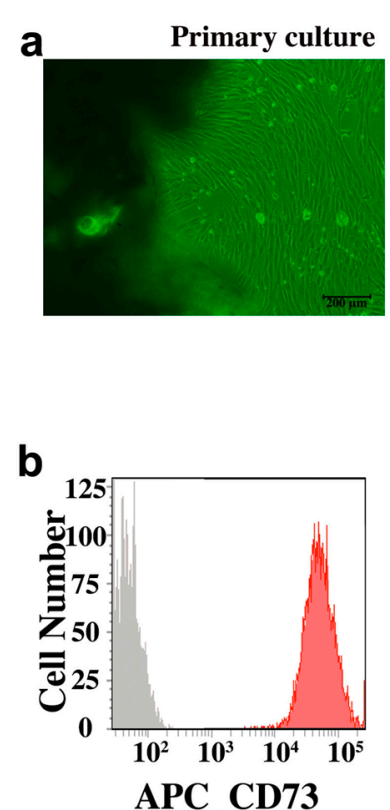

APC CD73

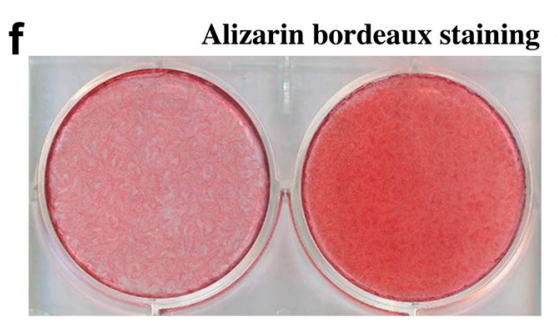

Control

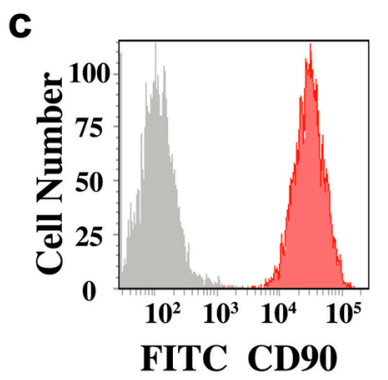

d
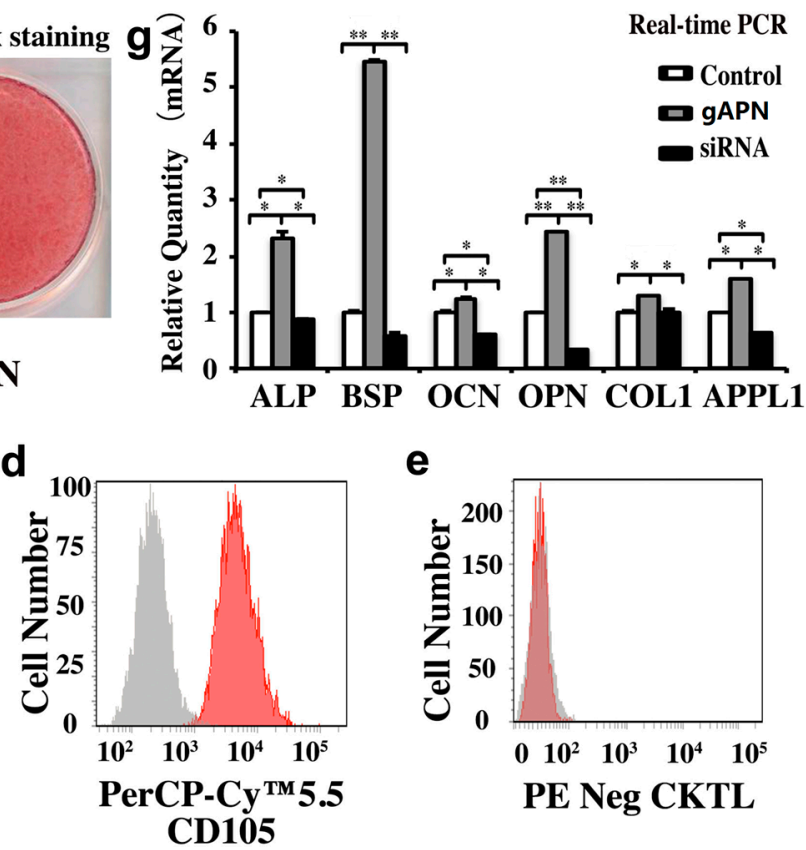

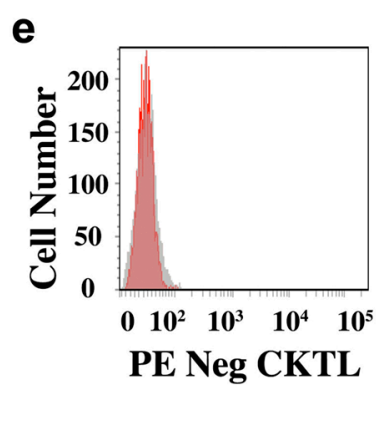

Figure 7. gAPN promoted the osteogenic commitment of h-JBMMSCs to favour osteoblastic differentiation. (a) The primary cultures were explanted from human granulation tissue in the 3rd molar extraction socket; (b-e) The primary cultured h-JBMMSCs were identified by flow cytometry. The h-JBMMSCs were positive for CD73, CD90, and CD105, but were negative for CD34, CD11b, CD19, CD45, and HLA-DR (red). All the results were with h-JBMMSC (red) and isotype control (light gray); (f) h-JBMMSCs were treated with vehicle and gAPN in osteogenesis induction medium for 21 days, followed by ascorbic acid staining; (g) Changes in gene expression of h-JBMMSCs following $24 \mathrm{~h}$ of gAPN treatment analysed using quantitative RT-PCR. Data are shown as the mean $\pm \mathrm{SD}$, with $n=4$. $* p<0.05$, ** $p<0.01$ as determined by one-way ANOVA.

\section{Discussion}

\subsection{Superimposition of CBCT (Cone Beam Computed Tomography) Models for the Location of Extraction Sockets}

Conventional X-ray [21], gravimetry [22], extraoral (casts) or intra-oral exams [23,24], optical projection [25], optical scanning [26], measurements on photographs [27], and bone mapping via sounding [24] cannot accurately pinpoint the 3D location of extraction sockets, because of artefacts including dimensional changes in impression and cast materials, access limitation, and saliva influence.

This is the first study to use CBCT superimposition to locate extraction sockets (see Figure 2a,b). $\mathrm{CBCT}$ scans are a mature 3D virtual tool for diagnosis, treatment planning, and pre-clinical simulations. One single scan by CBCT can provide not only detailed 2D images of the root socket structures in axial, coronal, and sagittal slices, but also a 3D reconstruction of the jaw. The 3D models of the extraction sockets were visible clearly and segmented easily from the CBCT scans. 3D models of the same extraction sockets in different time points could be superimposed using common stable landmarks as reference to evaluate the changes of extraction sockets $[28,29]$. We chose the cuspid of the canine and the first molar 
as the superimposition references, because the locations of these teeth are stable in mature beagles, and were not expected to change following the operation.

\subsection{Local Administration of gAPN Promoted Trabecular Bone Formation in Extraction Sockets}

After precisely locating the extraction sockets, we used $\mu \mathrm{CT}$ (see Figure 2) and histological analysis (see Figures 3-5) to analyse changes in the trabecular bone structure and mineral density following three months of scaffold placement loaded with gAPN or BMP2. We found that the gAPN and BMP2 scaffolds significantly increased TV/BV, Tb.Th, and Tb.N, and resulted in a decrease in the Tb.Sp separation (see Figure 2i). The control group exhibited decreased bone mineralisation and density, and enlarged bone marrow area, compared with the gAPN and BMP2 groups (see Figure 2i). Histological analyses of trabecular bone demonstrated the function of the gAPN and BMP2 scaffolds to increase the trabecular bone and reduce the marrow area in extraction sockets after 12 weeks compared with the control group (see Figures 3-5).

The Bio-Oss group exhibited the largest values of BV/TV, Tb, and Tb.N, as well as the smallest Tb.Sp (see Figure 2i); however, toluimide blue staining revealed the lowest formation of new bone in the BIO-Oss group (see Figure 4b). This was consistent with the results of Masson staining (see Figure 5b). In micro-CT experiments, all the mineralized bone could be detected, included anorganic bovine bone (Bio-Oss). This was the limitation of the micro-CT methodology. In the Bio-Oss group, the central regions were mainly occupied by mineral portions of bovine bone (Figure $3 \mathrm{~b}, \mathrm{f}$ ) and less new bone formation (Figures 4b, f and 5b,f). In the gAPN and BMP2 treatment groups, the implanted scaffold had been replaced by new bone (Figure $3 \mathrm{~g}$,h). So, the Bio-Oss group had more dense bone in micro-CT measurement. However, histology analysis experiments could distinguish the bone substitute and new bone formation. Compared with the Bio-Oss groups, the extraction sockets treated with scaffolds loaded with gAPN or BMP2 exhibited more toluidine blue (Figure 4) and Masson staining (Figure 5), clearly indicating the new bone formation in gAPN or BMP2 group. This is the reason that the trabecular separation is low (more dense) by CT evaluation while new bone formation is low by histology evaluation in Bio-Oss group. Our results are in line with previous studies that BMPs are potent osteoinductive factors [30], and can regulate the function and differentiation of cells involved in bone formation and healing [31,32]. This article is the first to report that scaffolds loaded with gAPN exhibit osteoinduction, which is consistent with the retarded growth of bone explants in APN-KO mice [33], and the observation that AdipoR1 mice exhibit increased bone volume and trabecular numbers than wild-type mice [11].

\subsection{Local Placement of Scaffold Loaded with gAPN Increased Osteoblast Differentiation and Collagen Type I Expression}

We explored changes in osteoblast-related protein expression by immunostaining socket slides of the different groups 12 weeks after scaffolds implantation. The gAPN and BMP2 groups exhibited significantly increased numbers of osteoblasts expressing OCN and BSP compared with the control (see Figure 6a,b, Table S1). However, the expression of collagen type I was lowest in the Bio-Oss group (see Figure 6c, Table S1), which is consistent with the lower intensity of toluidine blue and Masson staining in the Bio-Oss group (see Figures 4 and 5). These findings indicate that the scaffolds loaded with gAPN or BMP2 exhibited superior osteoinduction compared with Bio-Oss. 


\subsection{Osteogenic Differentiation of h-JBMMSCs Induced with gAPN in Vitro}

Bone remodelling is a delicate balance between bone resorption by osteoclasts and bone deposition by osteoblasts [34]. This equilibrium is influenced by differentiation and activity of osteoclasts and osteoblasts and [35]. To gain insight into the mechanisms underlying gAPN-induced oestrogenic differentiation of h-JBMMSCs, we investigated the expression of osteogenesis-related genes after $24 \mathrm{~h}$ of exposure to gAPN. We found that gAPN promoted osteogenic differentiation of the primary cultured h-JBMMSCs in vitro, which was accompanied by greater mineralisation deposition activity (see Figure 7c), and greater expression of osteoblast-related genes such as collagen type I, osteopontin (OPN), bone sialoprotein (BSP), and osteocalcin (OCN), and which was reversed by APPL1 siRNA (see Figure 7d). Many of the effects of APN in various cell types are known to be mediated by APPL1 [36,37], but this is the first demonstration of such a mechanism in h-JBMMSCs. APPL1 is involved in cell signalling by activating Akt, PI3-kinase (PI3K), the adiponectin receptor, and TrkA [38]. Previous studies have shown that PI3K [36], Akt [37], and TrkA [39] pathways affect osteoblast function via the proliferation and differentiation of BMSCs. More work is needed to investigate the involvement of APN and its signalling pathways in regulating h-JBMMSCs in vivo.

\section{Experimental Section}

\subsection{Recombinant $g A P N$}

The His-tagged C-terminal part of human APN (i.e., amino acids 106-244) was expressed in BL21 (DE3) bacterial cells. His-tagged gAPN was purified using GE Pharmacia AKTA Purifier 10 (Ramsey, MN, USA) followed by endotoxin removal (L00338, GenScript, Piscataway, NJ, USA) and Zeba Spin Desalting Columns (89893, Pierce, Rockford, IL, USA). The gAPN solution was filtrated to remove bacteria using a $0.22-\mu \mathrm{m}$ separating film.

\subsection{Materials}

Chitosan (with a molecular weight of $500 \mathrm{kDa}$ and a deacetylation grade of 90\%), span-80 and tripolyphosphate (TPP) were purchased from Beijing Chemical Reagents Company (Beijing, China). PLGA ( $\mathrm{nLA} / \mathrm{nGA}=50 / 50$ ) was obtained from the Jinan Daigang Bioengineering Co., Ltd. (Jinan, Shandong, China), and $\beta$-TCP was prepared in our laboratory (particle size $0.25-1 \mathrm{~mm}$ ). The other reagents were of analytical grade, and applied in accordance with the manufacturer's instructions. All chemicals were sterilised using cobalt- $60 \gamma$ radiation before use.

\subsection{Preparation of Scaffolds}

Chitosan microspheres were formulated using an emulsion-ionic modus [40]. In brief, $1 \mathrm{~mL}$ of $2 \%$ $(v / v)$ aqueous acetic acid with $1 \mathrm{mg}$ gAPN or $0.2 \mathrm{mg}$ BMP2 (Sigma-Aldrich, St. Louis, MO, USA) was added to a mixture, which was consist of $900 \mathrm{mg}$ of chitosan dissolved in $29 \mathrm{~mL}$ of $2 \%(\mathrm{v} / \mathrm{v})$ aqueous acetic acid. Then, the hybrid was put into $300 \mathrm{~mL}$ of liquid paraffin include $2 \%(w / v)$ of span-80 and mechanically mixed for $2 \mathrm{~h}$ (C-MAG HS 10, IKA ${ }^{\circledR}$ Works Inc., Wilmington, NC, USA). Next, 70 mL of $5 \%(w / v)$ TPP was put into the emulsion and mixed for $4 \mathrm{~h}$ at room temperature. Excess petroleum 
ether, isopropyl alcohol, and distilled water were used to wash the mixture repeatedly, then the microspheres were acquired with lyophilisation. Microsphere-scaffold composites were fabricated by thermal phase separation. Then, $12 \mathrm{~mL}$ of 1,4-dioxane was used to dissolve $720 \mathrm{mg}$ of PLGA, and $360 \mathrm{mg}$ of $\beta$-TCP was put into the mixture after mixing for $30 \mathrm{~min}$.

The $\beta$-TCP were dispersed by ultrasonication (Huanan Ultrasonic Equipment Co., Ltd., Guangzhou, China) for $10 \mathrm{~min}$. Next, $240 \mathrm{mg}$ of the chitosan-gAPN or chitosan-BMP2 microspheres was added to the solution, which was then stirred to completely disperse the microspheres and finally frozen using liquid nitrogen. The PLGA/ $\beta$-TCP/CMs scaffolds with and without APN or BMP2 were acquired after having been lyophilised according to the manufacturer's instruction (Labconco, Kansas City, MO, USA). The above procedures were conducted under aseptic conditions.

\subsection{Surgical Procedure}

We used male beagle dogs aged 14-18 months with body weights of 12-15 kg. All animals had intact permanent dentition and healthy periodontium. All the operations were done after a 2-week adaptation, as shown in Figure 8. Throughout the experiments, the dogs were fed once per day with soft food to prevent mechanical trauma during healing. All protocols involving the dogs met the guidelines described in the Association for Assessment and Accreditation of Laboratory Animal Care and were approved by the Peking University Health Centre Institutional Animal Care and Use Committee and Peking University Health Centre Ethnics Committee (LA2013-3, January 2013). One professional surgeon performed all operations.

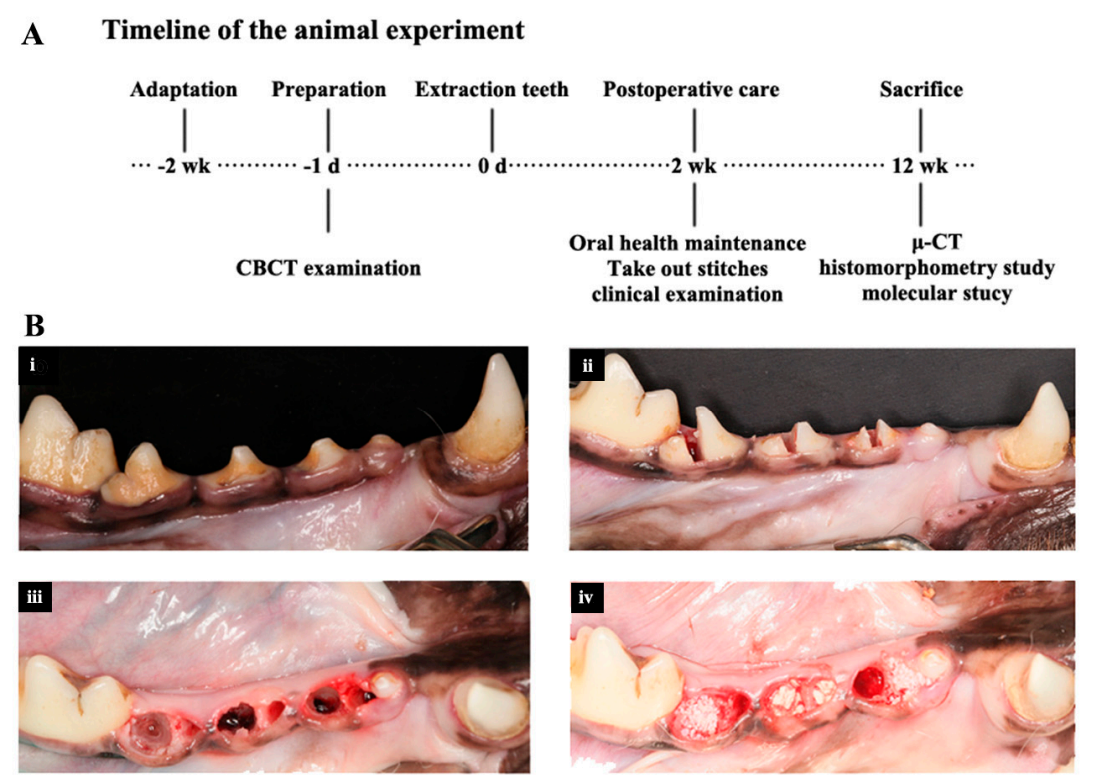

Figure 8. (A) Timeline of the treatment; (B) Treatment groups. (i) The 2nd, 3rd, and 4th premolar teeth before extraction; (ii) Teeth were segmented before extraction; (iii) Extraction sockets immediately following removal of the roots; extraction was accomplished with as little trauma to the alveolus as possible; (iv) The control, Bio-Oss, and scaffolds loaded with BMP2 or APN were randomly applied to the extraction sockets (lateral view of the left jaw). 
The 2nd, 3rd, and 4th premolar teeth in the unilateral mandible were extracted according to the protocol described in Reference [41]. During surgery, the animals were anaesthetised with intramuscularly administered atropine $(0.04 \mathrm{mg} / \mathrm{kg})$ and ketamine $(2.5 \mathrm{mg} / \mathrm{kg})$, as well as a $4 \%$ solution of intravenously administered pentobarbital sodium (25-30 mg/kg). Before extraction of the teeth, local anaesthesia with $2 \%$ xylocaine (1/80,000 epinephrine) was administrated. The same medications were used to obtain the pre-surgery $\mathrm{CBCT}$.

\subsection{Ridge Preservation}

The 12 fresh extraction sockets were randomly allocated to blank control (I), Bio-Oss $(0.25-1 \mathrm{~mm}$ particle size, Geistlich, Wolhusen, Switzerland) (II), scaffolds loaded with BMP2 (III) or APN (IV). The choice of the different treatments was determined using casting lots. Mucoperiosteal flaps were raised with periosteal-releasing incisions to make sure the wound could be closed without any tension, and then the wounds were closed with interrupted and mattress sutures, as shown in Figure 8. Antibiotic therapy (ampicillin sodium; $25 \mathrm{mg} / \mathrm{kg}$ intramuscularly daily) and analgesics (naproxen; $1.1 \mathrm{mg} / \mathrm{kg}$ taken orally) were administered for 5 days, and the sutures were removed 15 days after the surgery. The euthanasia was performed by anesthesia overdose 12 weeks after scaffolds implantation.

\subsection{CBCT Scans}

CBCT scans were taken 1 day prior to extraction of the teeth using a NewTom VG 3D Imaging System (NewTom Dental Inc., Verona, Italy) with a window of scan of $12 \mathrm{~cm} \times 8 \mathrm{~cm}$ and a voxel size of $0.15 \mathrm{~mm}$ and exported data using the Digital Imaging and Communications in Medicine (DICOM) format.

\section{7. $\mu$ CT Scans}

Radiographs of the trabecular bone architecture of dog mandibles were obtained using a high-resolution $\mu \mathrm{CT}$ system (Inveon, Siemens, Germany) following sacrifice after 12 weeks. The specimens were scanned at an operating voltage of $60 \mathrm{kV}$ and a current of $300 \mu \mathrm{A}$, with an effective pixel size of $8.5 \mu \mathrm{m}$. Data from the $\mu \mathrm{CT}$ scans were exported using DICOM format.

\subsection{Socket Location and $\mu C T$ Analysis}

Three-dimensional (3D) STL models of the premolars and 1st molars were segmented from the CBCT DICOM data prior to extraction of the teeth using Materialise Mimics 14.1 (an interactive medical image control system, Materialise Inc., Leuven, Belgium). The canines and 1st molars were segmented using the same software from the corresponding $\mu \mathrm{CT}$ DICOM files. The first molar and the canine were used as reference objects. The 3D STL models before extraction were superimposed and repositioned onto the 3D STL models from the $\mu \mathrm{CT}$ data using digital image process software (Geomagic Studio 2012, Raindrop Geomagic, Research Triangle Park, Durham, NC, USA) The repositioned 3D digital model prior to extraction, which contained information on the position of the extracted teeth, was used as a mask to help locate the extraction sockets, as shown in Figure 2c, and to locate the region of interest (ROI). A root-shaped region was dissected. The threshold of trabecular bone was the same for each 
specimen (as shown in Figure S1). Additionally, BV/TV, trabecular number (Tb.N), trabecular thickness, and Tb.Sp were analysed using software provided by Materialise, as shown in Figure $2 \mathrm{i}$.

\subsection{Histomorphometry Analysis}

$15 \%$ EDTA was used to demineralise sample. Then, the samples were processed by graded alcohols, xylene and paraffin. The thickness of slices was $4-\mu \mathrm{m}$. To quantify the new bone formation, three pieces of histological slices were stained with H\&E, toluidine blue, and Masson, and randomly selected and observed using an optical microscope at magnifications of 20 and 200.

\subsection{Immunohistochemical Labelling}

A molecular analysis of the bone regeneration process was carried out using immunohistochemistry 12 weeks after scaffolds implantation in the extraction sockets of Beagle dogs. For immunohistochemistry, all tissue slices were deplasted and treated with antibodies to determine the expression of collagen type I (Col-1) (1:100, Santa Cruz, CA, USA), osteocalcin (OCN) (1:100, Santa Cruz, CA, USA), and bone sialoprotein (BSP) (1:100, Santa Cruz, CA, USA).

\subsection{Numerical Evaluation of the Histology}

The results of immunohistochemistry staining were semiquantitatively valued by two researchers. The researchers did not know the details of specimens. The intensity of staining (strong, moderate, weak, and negative) was graded as 4, 3, 2 and 1, respectively. The final labeling index obtained by the previous intensity results multiplied the percentage of positive cells $(0,25 \%, 50 \%$ or $75 \%)$.

\subsection{Donor Socket Granulation Tissue and Primary h-JBMMSCs Cultures}

Human mandible third molars were extracted and the granulation bone marrow was gained from the socket. Primary h-JBMMSCs were established from the granulation tissue explants of donors using a method described previously [20]. In brief, the granulation tissue was minced and placed separately into the 12-well plates. The cell culture medium was changed per 2-3 days following the emergence of outgrowth. The cells were cultured in medium consisting of $\alpha$-MEM (Life Technologies, Rockville, MD, USA), 15\% foetal bovine serum (FBS, Life Technologies, Rockville, MD, USA), $100 \mu \mathrm{M}$ of L-ascorbic acid 2-phosphate (WAKO, Tokyo, Japan), $2 \mathrm{mM}$ of L-glutamine (Life Technologies), $100 \mathrm{U} / \mathrm{mL}$ of penicillin, and $100 \mu \mathrm{g} / \mathrm{mL}$ of streptomycin (Sigma-Aldrich, St. Louis, MO, USA) at $37{ }^{\circ} \mathrm{C}$ in $5 \% \mathrm{CO}_{2}$. The study protocol was documented by the Medical Ethical Commission of the Peking University School and Hospital of Stomatology, Beijing, People's Republic of China (PKUSSIRB-201520026). All patients provided informed consent. Human tissue samples were handled according to the tenets of the Declaration of Helsinki. 


\subsection{Flow Cytometry}

The cells were first dissociated and followed by process with the antibodies: FITC Mouse Anti-Human CD90 (BD); APC Mouse Anti-Human CD73 (BD); PerCP-Cy ${ }^{\mathrm{TM}} 5.5$ Mouse Anti-Human CD105 (BD); PE hMSC Negative Cocktail including CD34 PE, CD11b PE, CD19 PE, CD45 PE, HLA-DR PE (BD); and the respective isotype-matched negative control antibodies on ice for $30 \mathrm{~min}$. The cells were analysed using a MACSQuant Analyzer and the FlowJo software package (Tree Star, Ashland, OR, USA) were used to assess the data.

\subsection{4. h-JBMMSCs Osteogenic Induction}

Cells from the fourth passage were choosed to conduct the experiments. To investigate cell osteogenic differentiation, cells were seeded at $2 \times 10^{4}$ per well in 12-well plates. After the cultures reached confluence, the medium was changed to DMEM with $2 \%(v / v)$ KnockOut ${ }^{\mathrm{TM}}$ Serum Replacement, $100 \mathrm{U} / \mathrm{mL}$ penicillin, and $100 \mathrm{mg} / \mathrm{mL}$ streptomycin containing 10-nM dexamethasone, 10-mM $\beta$-glycerophosphate, and $50-\mu \mathrm{g} / \mathrm{mL}$ L-ascorbic acid (Sigma-Aldrich, St. Louis, MO, USA). The cells were then further cultured for 21 days with or without $1-\mu \mathrm{g} / \mathrm{mL}$ APN.

\subsection{Knockdown of APPL1 in h-JBMMSCs}

siRNA sequences (Santa Cruz, CA, USA) designed for the knockdown of human APPL1 were tested with h-JBMMSCs. Because it is difficult to transfect stem cells, Lipofectamine ${ }^{\circledR} 2000$ Transfection Reagent was used. The h-JBMMSCs reached 30\%-50\% confluence in 6-well plates, and transfected with 100-nM scrambled or APPL1 siRNA for $6 \mathrm{~h}$ using Lipofectamine ${ }^{\circledR} 2000$ Transfection Reagent. The siRNA sequence was GCUUAGUUCUUGUCAUGCAtt. APN treatment commenced $48 \mathrm{~h}$ post-transfection, and the APPL1 knockdown efficiency was assessed using the real-time PCR described below.

\subsection{Quantitative Real-Time PCR}

TRIzol (Invitrogen, Carlsbad, CA, USA) was used to extract sample total RNA. SuperScript III reverse transcriptase (Life Technologies) and oligo(dT)20 primer (Life Technologies) were used to generate the first strand of cDNA. Power SYBR Green PCR Master Mix (Applied Biosystems) and a 7500 Real-Time PCR System (Applied Biosystems) were used to perform Real-time PCR. $\beta$-actin served as a control. The sequence of primers was as follows: human $\beta$-actin sense 5'-CAAG GCCAACCGCGAGAAGATGAC-3', antisense 5'-GCCAGAGGCGTACAGGGATAGCACA-3'; ALP sense 5'-CCCGCTTTAACCAGTGCAAC-3', antisense 5'-GAGCTGCGTAGCGATGTCC-3'; BSP sense 5'-GGCCACGATATTATCTTTACAAGC-3', antisense 5'-CCTCTTCTGAACTGTCATCTCCA-3'; Col-1 sense 5'-GAGGGCCAAGACGAAGACATC-3', antisense 5'-CAGATCACGTCATCGCACAAC-3'; OCN sense 5'-GGCGCTACCTGTATCAATGG-3', antisense 5'-GTGGTCAGCCAACTCGTCA-3'; OPN sense 5'-GAAGTTTCGCAGACCTGACAT-3', and antisense 5'-GTATGCACCATTCAACTCCTCG-3'. 


\title{
4.17. Statistical Analysis
}

Data were shown as the mean \pm standard deviation (SD). One-way analysis of variance (ANOVA) was used to evaluated the statistically significant differences $(p<0.05)$ among the various groups. SPSS 19.0 software package (SPSS Inc., Chicago, IL, USA) was used for all statistical analysis.

\section{Conclusions}

We conducted an in vivo investigation of the extraction sockets of beagles, and found that composite scaffold loaded with APN exhibited superior activity for guided bone regeneration compared with blank control. In vitro experiments revealed that $1 \mu \mathrm{g} / \mathrm{mL}$ of gAPN can induce osteoblastic differentiation in human h-JBMMSCs. The composite scaffold with APN has significant potential for clinical applications in bone tissue engineering.

\section{Supplementary Materials}

Supplementary materials can be found at http://www.mdpi.com/1422-0067/16/10/24946/s1.

\section{Acknowledgments}

We want to thank Yi Deng for his assistance with preparation of this manuscript.

This work was supported by the National Natural Science Foundation of China, grant number 81300851, which was awarded to Yuwei Wu, and the Beijing Municipal Natural Science Foundation, Grant number SKLODd2009001, which was awarded to Zhihui Tang. Peking University School of Stamotology Youth Fund to Yinfei Pu.

\section{Author Contributions}

Conceived and designed the experiments: Yuwei Wu and Zhihui Tang; Performed the experiments: Hongcheng $\mathrm{Hu}$, Yinfei Pu, Songhe Lu, Yuan Guo, Hui Lu, Deli Li, Xuefen Li; Analyzed the data: Hongcheng Hu, Yinfei Pu; Contributed reagents/materials/analysis tools: Kuo Zhang, Zichen Li; Wrote the paper: Yuwei $\mathrm{Wu}$, Hongcheng $\mathrm{Hu}$.

\section{Conflicts of Interest}

The authors declare no conflict of interest.

\begin{abstract}
Abbreviations
gAPN: globular adiponectin; PLGA: poly (L-lactic-co-glycolic) acid; $\beta$-TCP: $\beta$-tricalcium phosphate; CBCT: cone beam computed tomography; BMP-2: bone morphogenetic protein 2; h-JBMMSCs: human jaw bone marrow mesenchymal stem cells; Tb: trabecular bone; BV: bone volume; TV: tissue volume; Tb.Th: trabecular thickness; Tb.N: trabecular number; Tb.Sp: trabecular separation; OCN: osteocalcin; OPN: osteopontin; BSP: bone sialoprotein; Col-1: Collagen Type 1; BMSCs: bone marrow mesenchymal
\end{abstract}


stem cells; TPP: tripolyphosphate; ROI: region of interest; FBS: foetal bovine serum; SiRNA: small interfering RNA.

\section{References}

1. Wu, Y.W.; Lu, H.; Hu, H.C.; Guo, Y.; Li, D.; Li, Z.C.; Tang, Z.H. Preparation and in vitro release behavior of adiponectin microsphere-scaffold composite. J. Pract. Stomatol. 2015, 31, 171-174.

2. Wu, Y.W.; Lu, H.; Hu, H.C.; Guo, Y.; Li, D.; Li, Z.C.; Tang, Z.H. The effect of local delivery of adiponectin from biodegradable microsphere-scaffold composite on new bone formation in adiponectin knockout mice. J. Colloid Interface Sci. 2015, Revision.

3. Wang, C.K.; Ho, M.L.; Wang, G.J.; Chang, J.K.; Chen, C.H.; Fu, Y.C.; Fu, H.H. Controlled-release of rhBMP-2 carriers in the regeneration of osteonecrotic bone. Biomaterials 2009, 30, 4178-4186.

4. Benglis, D.; Wang, M.Y.; Levi, A.D. A comprehensive review of the safety profile of bone morphogenetic protein in spine surgery. Neurosurgery 2008, 62, ONS423-ONS431.

5. Chen, N.F.; Smith, Z.A.; Stiner, E.; Armin, S.; Sheikh, H.; Khoo, L.T. Symptomatic ectopic bone formation after off-label use of recombinant human bone morphogenetic protein-2 in transforaminal lumbar interbody fusion. J. Neurosurg. Spine 2010, 12, 40-46.

6. Tsao, T.S.; Lodish, H.F.; Fruebis, J. ACRP30, a new hormone controlling fat and glucose metabolism. Eur. J. Pharmacol. 2002, 440, 213-221.

7. Pajvani, U.B.; Du, X.; Combs, T.P.; Berg, A.H.; Rajala, M.W.; Schulthess, T.; Engel, J.; Brownlee, M.; Scherer, P.E. Structure-function studies of the adipocyte-secreted hormone Acrp30/adiponectin. Implications fpr metabolic regulation and bioactivity. J. Biol. Chem. 2003, 278, 9073-9085.

8. Padmalayam, I.; Suto, M. Role of adiponectin in the metabolic syndrome: Current perspectives on its modulation as a treatment strategy. Curr. Pharm. Des. 2013, 19, 5755-5763.

9. Villarreal-Molina, T.; Posadas-Romero, C.; Romero-Hidalgo, S.; Antunez-Arguelles, E.; Bautista-Grande, A.; Vargas-Alarcon, G.; Kimura-Hayama, E.; Canizales-Quinteros, S.; Juarez-Rojas, J.G.; Posadas-Sanchez, R.; et al. The ABCA1 gene R230C variant is associated with decreased risk of premature coronary artery disease: The genetics of atherosclerotic disease (GEA) study. PLoS ONE 2012, 7, e49285.

10. Yu, L.; Tu, Q.; Han, Q.; Zhang, L.; Sui, L.; Zheng, L.; Meng, S.; Tang, Y.; Xuan, D.; Zhang, J.; et al. Adiponectin regulates bone marrow mesenchymal stem cell niche through a unique signal transduction pathway: An approach for treating bone disease in diabetes. Stem Cells 2015, 33, 240-252.

11. Lin, Y.Y.; Chen, C.Y.; Chuang, T.Y.; Lin, Y.; Liu, H.Y.; Mersmann, H.J.; Wu, S.C.; Ding, S.T. Adiponectin receptor 1 regulates bone formation and osteoblast differentiation by GSK-3 $\beta / \beta$-catenin signaling in mice. Bone 2014, 64, 147-154.

12. Luo, X.H.; Guo, L.J.; Yuan, L.Q.; Xie, H.; Zhou, H.D.; Wu, X.P.; Liao, E.Y. Adiponectin stimulates human osteoblasts proliferation and differentiation via the MAPK signaling pathway. Exp. Cell Res. 2005, 309, 99-109.

13. Oshima, K.; Nampei, A.; Matsuda, M.; Iwaki, M.; Fukuhara, A.; Hashimoto, J.; Yoshikawa, H.; Shimomura, I. Adiponectin increases bone mass by suppressing osteoclast and activating osteoblast. Biochem. Biophys. Res. Commun. 2005, 331, 520-526. 
14. Mitsui, Y.; Gotoh, M.; Fukushima, N.; Shirachi, I.; Otabe, S.; Yuan, X.; Hashinaga, T.; Wada, N.; Mitsui, A.; Yoshida, T.; et al. Hyperadiponectinemia enhances bone formation in mice. BMC Musculoskelet. Disord. 2011, 12, 18.

15. Lee, H.W.; Kim, S.Y.; Kim, A.Y.; Lee, E.J.; Choi, J.Y.; Kim, J.B. Adiponectin stimulates osteoblast differentiation through induction of COX2 in mesenchymal progenitor cells. Stem Cells 2009, 27, 2254-2262.

16. Wu, Y.; Tu, Q.; Valverde, P.; Zhang, J.; Murray, D.; Dong, L.Q.; Cheng, J.; Jiang, H.; Rios, M.; Morgan, E.; et al. Central adiponectin administration reveals new regulatory mechanisms of bone metabolism in mice. Am. J. Physiol. Endocrinol. Metab. 2014, 306, E1418-E1430.

17. Bouxsein, M.L.; Boyd, S.K.; Christiansen, B.A.; Guldberg, R.E.; Jepsen, K.J.; Muller, R. Guidelines for assessment of bone microstructure in rodents using micro-computed tomography. J. Bone Miner. Res. 2010, 25, 1468-1486.

18. Huang, C.Y.; Lee, C.Y.; Chen, M.Y.; Tsai, H.C.; Hsu, H.C.; Tang, C.H. Adiponectin increases BMP-2 expression in osteoblasts via AdipoR receptor signaling pathway. J. Cell. Physiol. 2010, $224,475-483$.

19. Nudelman, F.; Pieterse, K.; George, A.; Bomans, P.H.; Friedrich, H.; Brylka, L.J.; Hilbers, P.A.; de With, G.; Sommerdijk, N.A. The role of collagen in bone apatite formation in the presence of hydroxyapatite nucleation inhibitors. Nat. Mater. 2010, 9, 1004-1009.

20. Nakajima, R.; Ono, M.; Hara, E.S.; Oida, Y.; Shinkawa, S.; Pham, H.T.; Akiyama, K.; Sonoyama, W.; Maekawa, K.; Kuboki, T. Mesenchymal stem/progenitor cell isolation from tooth extraction sockets. J. Dent. Res.2014, 93, 1133-1140.

21. Alpiste-Illueca, F. Dimensions of the dentogingival unit in maxillary anterior teeth: A new exploration technique (parallel profile radiograph). Int. J. Periodontics Restor. Dent. 2004, 24, 386-396.

22. Proussaefs, P.T.; Valencia, G.; Lozada, J.; Tatakis, D.N. A method to assess the clinical outcome of ridge augmentation procedures. J. Periodontol. 2002, 73, 302-306.

23. Cardaropoli, G.; Lekholm, U.; Wennstrom, J.L. Tissue alterations at implant-supported single-tooth replacements: A 1-year prospective clinical study. Clin. Oral Implant. Res. 2006, 17, 165-171.

24. Chen, L.C.; Lundgren, T.; Hallstrom, H.; Cherel, F. Comparison of different methods of assessing alveolar ridge dimensions prior to dental implant placement. J. Periodontol. 2008, 79, 401-405.

25. Studer, S.P.; Lehner, C.; Bucher, A.; Scharer, P. Soft tissue correction of a single-tooth pontic space: A comparative quantitative volume assessment. J. Prosthet. Dent. 2000, 83, 402-411.

26. Jemt, T.; Lekholm, U. Single implants and buccal bone grafts in the anterior maxilla: Measurements of buccal crestal contours in a 6-year prospective clinical study. Clin. Implant Dent. Relat. Res. 2005, 7, 127-135.

27. Ricci, A. An objective method to measure soft tissue behavior around single-tooth implants. Part 1: Vertical measurements. Eur. J. Esthet. Dent. 2007, 2, 406-418.

28. Grauer, D.; Cevidanes, L.S.; Proffit, W.R. Working with DICOM craniofacial images. Am. J. Orthod. Dentofac. Orthop. 2009, 136, 460-470.

29. Terajima, M.; Yanagita, N.; Ozeki, K.; Hoshino, Y.; Mori, N.; Goto, T.K.; Tokumori, K.; Aoki, Y.; Nakasima, A. Three-dimensional analysis system for orthognathic surgery patients with jaw deformities. Am. J. Orthod. Dentofac. Orthop. 2008, 134, 100-111. 
30. Fisher, D.M.; Wong, J.M.; Crowley, C.; Khan, W.S. Preclinical and clinical studies on the use of growth factors for bone repair: A systematic review. Curr. Stem Cell Res. Ther. 2013, 8, 260-268.

31. Bessa, P.C.; Casal, M.; Reis, R.L. Bone morphogenetic proteins in tissue engineering: The road from the laboratory to the clinic, part I (basic concepts). J. Tissue Eng. Regen. Med. 2008, 2, 1-13.

32. Wozney, J.M.; Rosen, V. Bone morphogenetic protein and bone morphogenetic protein gene family in bone formation and repair. Clin. Orthop. Relat. Res. 1998, 346, 26-37.

33. Tu, Q.; Zhang, J.; Dong, L.Q.; Saunders, E.; Luo, E.; Tang, J.; Chen, J. Adiponectin inhibits osteoclastogenesis and bone resorption via APPL1-mediated suppression of Akt1. J. Biol. Chem. 2011, 286, 12542-12553.

34. Martin, T.J.; Sims, N.A.; Ng, K.W. Regulatory pathways revealing new approaches to the development of anabolic drugs for osteoporosis. Osteoporos. Int. 2008, 19, 1125-1138.

35. Raisz, L.G. Physiology and pathophysiology of bone remodeling. Clin. Chem. 1999, 45, 1353-1358.

36. Kim, E.K.; Lim, S.; Park, J.M.; Seo, J.K.; Kim, J.H.; Kim, K.T.; Ryu, S.H.; Suh, P.G. Human mesenchymal stem cell differentiation to the osteogenic or adipogenic lineage is regulated by AMP-activated protein kinase. J. Cell. Physiol. 2012, 227, 1680-1687.

37. Kanazawa, I.; Yamaguchi, T.; Yano, S.; Yamauchi, M.; Yamamoto, M.; Sugimoto, T. Adiponectin and AMP kinase activator stimulate proliferation, differentiation, and mineralization of osteoblastic MC3T3-E1 cells. BMC Cell Biol. 2007, 8, 51.

38. Tan, Y.; You, H.; Coffey, F.J.; Wiest, D.L.; Testa, J.R. Appl1 is dispensable for Akt signaling in vivo and mouse T-cell development. Genesis 2010, 48, 531-539.

39. Mikami, Y.; Suzuki, S.; Ishii, Y.; Watanabe, N.; Takahashi, T.; Isokawa, K.; Honda, M.J. The p75 neurotrophin receptor regulates MC3T3-E1 osteoblastic differentiation. Differ. Res. Biol. Divers. 2012, 84, 392-399.

40. Wang, L.; Zheng, L.; Li, C.; Dong, S.; A, L.; Zhou, Y. Adrenomedullin delivery in microsphere-scaffold composite for remodeling of the alveolar bone following tooth extraction: An experimental study in the rat. Biomed. Eng. Online 2013, 12, 99.

41. Verstraete, F.J. Instrumentation and technique of removal of permanent teeth in the dog. J. S. Afr. Vet. Assoc. 1983, 54, 231-238.

(C) 2015 by the authors; licensee MDPI, Basel, Switzerland. This article is an open access article distributed under the terms and conditions of the Creative Commons Attribution license (http://creativecommons.org/licenses/by/4.0/). 